\title{
A Case of Severe Panuveitis Associated with Psoriasis Vulgaris Successfully Treated with Infliximab
}

\author{
Yuri Sakurai $^{\mathrm{a}}$ Kenichi Namba ${ }^{\mathrm{a}}$ Kazuomi Mizuuchi $^{\mathrm{a}}$ \\ Toshihumi Nomurab ${ }^{\mathrm{b}}$ Susumu Ishida ${ }^{\mathrm{a}}$ \\ Departments of ${ }^{a}$ Ophthalmology and ${ }^{b}$ Dermatology, Hokkaido University Graduate \\ School of Medicine, Sapporo, Japan
}

\section{Key Words}

Uveitis · Psoriasis · Neovascularization · Infliximab

\begin{abstract}
Purpose: Uveitis associated with psoriasis vulgaris is usually seen as an anterior segment inflammation, and it is very rare that the inflammation extends to the posterior segment. We herein report a case of severe panuveitis associated with psoriasis vulgaris presenting as retinal neovascularization, leading to vitreous hemorrhages that were successfully treated with infliximab (IFX). Case Report: A 27-year-old male with psoriasis vulgaris was referred to our hospital due to prolonged severe uveitis OU. He showed a severe anterior chamber inflammation with fibrin formation and total posterior iris synechia OU. With topical corticosteroid treatment, these conditions were relieved for a short time; however, the intraocular inflammation was exacerbated with vitreous hemorrhages caused by retinal neovascularization OS. After the administration of IFX therapy, the intraocular inflammation and retinal neovascularization was resolved, and so far, no severe recurrences have been seen for 3 years with the therapy. Conclusion: When we see patients with severe panuveitis associated with psoriasis extending to the posterior segment, IFX treatment may be a good therapeutic option.


Sakurai et al.: A Case of Severe Panuveitis Associated with Psoriasis Vulgaris Successfully Treated with Infliximab

\section{Introduction}

Uveitis associated with psoriasis vulgaris is usually seen as an anterior segment inflammation, and it is very rare that the inflammation extends to the posterior segment [1, 2]. Our patient experienced panuveitis that was associated with psoriasis vulgaris and was accompanied by retinal vasculitis with retinal neovascularization leading to repeated vitreous hemorrhages. Infliximab (IFX) treatment was effective in resolving these issues.

\section{Case Presentation}

A 27-year-old male visited an ophthalmology clinic with a complaint of blurred vision OU. He showed bilateral uveitis that had been controlled with eye drops; however, it later exacerbated, and he was referred to our hospital.

He had suffered from psoriasis vulgaris since he was 14 years old and had been treated with topical corticosteroids and phototherapy by dermatologists; however, he had ceased all treatments on his own accord 2 years earlier. Since then, his skin condition had become worse due to the lack of medication.

At the first visit, the best-corrected visual acuity (BCVA) was $0.5 \mathrm{OD}$ and counting fingers OS. Intraocular pressure was $13 \mathrm{~mm} \mathrm{Hg} \mathrm{OD} \mathrm{and} 5 \mathrm{~mm} \mathrm{Hg} \mathrm{OS.} \mathrm{A} \mathrm{slit-lamp} \mathrm{examination}$ showed ciliary injections and 3+ flare and 3+ cells, fibrin formation in the anterior chamber with total posterior iris synechia OU, and fundi were invisible OU (fig. 1).

Besides psoriasis, other extraocular lesions such as oral aphthous ulcers or genital ulcers, or symptoms for Behçet's disease were not found. The patient's psoriasis was very severe; the skin over the whole body including the face and eyelids formed erythroderma and thick desquamation. Screening blood and urine tests did not show any positive results indicating sarcoidosis or other uveitis.

He underwent dexamethasone conjunctival injections, and topical treatments with $0.1 \%$ betamethasone eye drops and mydriatic eye drops were applied every hour. For psoriasis, corticosteroid ointment and ultraviolet irradiation were promptly started. With these medications, the anterior segment intraocular inflammation was reduced, and BCVA improved to $1.2 \mathrm{OD}$ and $0.8 \mathrm{OS} 3$ months later; however, the posterior synechia was not removed and fundi were still invisible OU. Ten months later, the patient underwent cataract surgery, and the posterior synechia was removed OS. After surgery, the vitreous opacity was mild, and the BCVA improved to 0.9 OS. However, the anterior chamber inflammation suddenly exacerbated with a vitreous hemorrhage OS 1 month postoperatively. The fundus was too blurred to be seen in detail; however, fluorescein angiography revealed marked leakage suggestive of retinal neovascularization (fig. 2). Since the patient developed further vitreous hemorrhages with a recurrence of anterior uveitis with his psoriasis not controlled well, IFX was administered 19 months following the first visit.

IFX ( $5 \mathrm{mg} / \mathrm{kg}$ ) was infused 2 and 6 weeks following the first infusion and every 8 weeks thereafter. After the IFX administration, a new vitreous hemorrhage occurred only once; however, it quickly disappeared and has not recurred. During the 3 years of IFX therapy, no severe recurrences were seen, and cataract surgery could be performed safely without postoperative inflammations OD. The final BCVA was $1.2 \mathrm{OD}$ and $0.8 \mathrm{OS}$. The patient's psoriasis was also gradually improved. 
Sakurai et al.: A Case of Severe Panuveitis Associated with Psoriasis Vulgaris Successfully Treated with Infliximab

\section{Discussion}

Uveitis associated with psoriasis vulgaris is mostly seen as acute anterior uveitis [1, 2]. Although a case of panretinal vasculitis with cystoid macular edema has already been reported [3], to the best of our knowledge, this is the first report of retinal vasculitis with retinal neovascularization leading to vitreous hemorrhages associated with psoriasis. We considered other causes of uveitis including sarcoidosis or Behçet's disease; however, all of them were excluded because of negative results of systemic examinations and no signs of extraocular symptoms related to these diseases. Because the patient's uveitis exacerbated along with the exacerbation of psoriasis, we diagnosed this case as psoriasis-associated uveitis. It is speculated that the systemic inflammatory disease in this patients was so severe as to induce inflammation in the posterior segment in addition to anterior segment.

This case was so severe that vitreous hemorrhages recurred frequently, even with topical corticosteroid treatment. However, after the IFX therapy, the ocular inflammation was relieved and has never recurred. Moreover, cataract surgery could be performed under the IFX treatment without severe postoperative inflammation.

IFX is a chimeric monoclonal antibody to tumor necrosis factor- $\alpha$ and has shown excellent efficacies in its worldwide use in the treatment of various immunological diseases [4, 5] including rheumatoid arthritis [4, 6] and Crohn's disease [5]. In psoriasis vulgaris, IFX is now available for severe cases that are refractory to existing treatments $[7,8]$.

Some uveitis cases with psoriasis that have undergone IFX treatment have been reported. Asukata et al. [9] reported a case of intractable anterior uveitis; Huynh et al. [10] reported 2 panuveitis cases, 1 anterior uveitis case and 1 scleritis case, and Goto et al. [3] reported a case showing retinal vasculitis with macular edema. In all cases, there was a remission of ocular inflammation, and skin lesions were well controlled with IFX administration. Our case presented a more severe panuveitis with inflammatory retinal neovascularization; however, IFX was effective in controlling the severe uveitis. Uveitis with psoriasis seems to be a good indication for the administration of IFX.

\section{Statement of Ethics}

This case report has been approved by the Ethics Committee of Hokkaido University Hospital, Sapporo, Japan.

\section{Disclosure Statement}

The authors have no financial interest to disclose in this work.

\section{References}

Lambert JR, Wright V: Eye inflammation in psoriatic arthritis. Ann Rheum Dis 1976;35:354-356.

Knox DL: Psoriasis and intraocular inflammation. Trans Am Ophthalmol Soc 1979;127:270.

Goto M, Ujihara H, Matsubara M: A case of psoriatic panuveitis treated by systemic infliximab. Jpn J Clin Ophtalmol 2010;64:1165-1168.

4 Maini R, St Clair EW, Breedveld F, Furst D, Kalden J, Weisman M, Smolen J, Emery P, Harriman G, Feldmann M, Lipsky P: Infliximab (chimeric anti-tumour necrosis factor alpha monoclonal antibody) versus placebo in rheumatoid arthritis patients receiving concomitant methotrexate: a randomised phase III trial. ATTRACT Study Group. Lancet 1999;354:1932-1939. 
Case Reports in

Ophthalmology

\begin{tabular}{l|l}
\hline Case Rep Ophthalmol 2016;7:191-194 \\
\hline DOI: 10.1159/000445285 & $\begin{array}{l}\text { ○ 2016 The Author(s). Published by S. Karger AG, Basel } \\
\text { www.karger.com/cop }\end{array}$ \\
\hline
\end{tabular}

Sakurai et al.: A Case of Severe Panuveitis Associated with Psoriasis Vulgaris Successfully Treated with Infliximab

-5 Hanauer S, Feagan BG, Lichtenstein GR, Mayer LF, Schreiber S, Colombel JF, Rachmilewitz D, Wolf DC, Olson A, Bao W, Rutgeerts P: Maintenance infliximab for Crohn's disease: the ACCENT I randomised trial. Lancet 2002;359:1541-1549.

6 Elliott MJ, Maini RN, Feldmann M, Long-Fox A, Charles P, Bijl H, Woody JN: Repeated therapy with monoclonal antibody to tumor necrosis factor alpha (cA2) in patients rheumatoid arthritis. Lancet 1994;344:1125-1127.

7 Christian A, Bernhard M: Infliximab for psoriasis and psoriatic arthritis. Clin Exp Rheumatol 2002;20:122125.

8 Chaudhari U, Romano P, Mulcahy LD, Dooley LT, Baker DG, Gottlieb AB: Efficacy and safety of infliximab monotherapy for plaque-type psoriasis: a randomized trial. Lancet 2001;351:1842-1847.

9 Asukata Y, Kawagoe T, Ishihara M, Nishida T, Nakamura S, Hayashi K, Mizuki N: Systemic infliximab was effective in a case of uveitis with psoriatic arthritis (in Japanese). Jpn J Clin Ophtalmol 2011;65:1117-1121.

-10 Huynh N, Cervantes-Castaneda RA, Bhat P, Gallagher MJ, Foster CS: Biological response modifier therapy for psoriatic ocular imflammatory disease. Ocul Immunol Inflamm 2008;16:89-93.
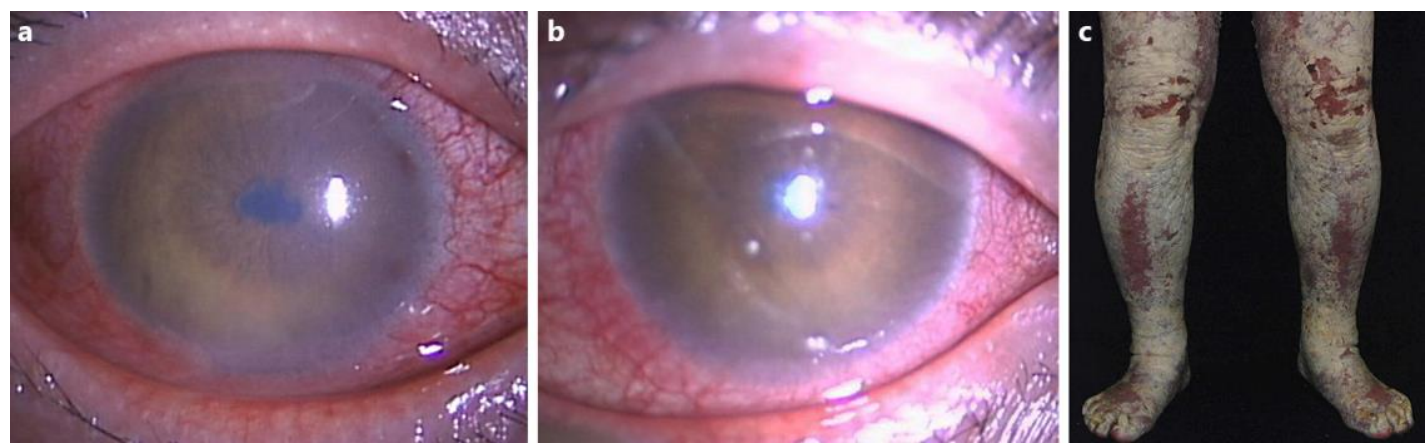

Fig. 1. Clinical findings during the initial visit. Ciliary injections and severe anterior chamber inflammations, such as 3+ flare and 3+ cells, and fibrin formation combined with total posterior synechia, were seen in OD (a) and OS (b). Erythroderma and thick desquamation were seen in the lower limbs (c).


Fig. 2. Before the IFX treatment, the ocular fundus was too blurred to be seen in detail due to a vitreous hemorrhage (a), and fluorescein angiography showed a marked leakage suggestive of retinal neovascularization (b). 